\title{
Diseño de un plan estratégico para las pymes del sector de bares y expendio de bebidas alcohólicas de la localidad de Chapinero, Bogotá, para incrementar su competitividad y perdurabilidad
}

pags $95-110$

Grupo de Investigación: Centro de Investigación y Desarrollo Empresarial CINDE Línea de investigación: Emprendimiento y empresarialismo Martín Cuevas• \& Rafael Avella••

Recibido: 5 de junio de 2015

\section{RESUMEN}

El Proyecto presenta la descripción de los procesos de implementación de un plan estratégico, para el sector económico de bares, tomando en cuenta hallazgos encontrados en el sector y resaltando las mejores estrategias de algunos establecimientos. Así mismo, se analizaron algunas problemáticas presentes en los bares, con el fin de encontrar los principales focos de riesgo que los afecten, en la búsqueda de perdurabilidad y competitividad.

El producto final es el diseño de un plan estratégico, de acuerdo a los requerimientos del sector de bares en la localidad de Chapinero Bogotá. Este comprende diferentes fuerzas de afección del entorno de los centros de entretenimiento, al nivel de la localidad de Chapinero. Además, la inclusión de estrategias comprendidas por los hallazgos más relevantes, que a su vez sirvan para la gestión, tanto de nuevos bares, como de los ya existentes.

Palabras clave: Plan Estratégico, Estudio de Mercados, Estudio Técnico, Estudio Administrativo.

\section{Aceptado: 12 de junio de 2015}

\footnotetext{
- Especialista en Administración. Docente investigador. Fundación Universidad de América. martin.cuevas@investigadores. uamerica.edu.co

-. Estudiante coinvestigador, Ingeniería Industrial Fundación Universidad de América.
} 


\section{INTRODUCCIÓN}

El crecimiento económico de la nación, tiende a dar gran protagonismo a las pequeñas y medianas empresas, puesto que son estas, las que llegan a dar en importante medida sostenimiento económico al país, fuente de empleo, y entornos empresariales dinámicos y atractivos. Además, se ha implementado en los estudiantes del programa de ingeniería industrial, no solo la idea de fomentar negocios de producción, sino también la creación de organizaciones enfocadas a los servicios.

Según Diana Carolina Cantillo periodista del periódico El Espectador, en el año 2011,

"En Colombia el $96 \%$ de las empresas son Mipymes y Bogotá concentra el mayor número de éstas con el $96,4 \%$ de las 23.000 existentes. Por eso la capital colombiana fue el epicentro de la II Feria Internacional de las Mipymes, organizada por ACOPI regional Bogotá-Cundinamarca, donde el $76 \%$ del empleo lo generan las micro, pequeñas y medianas empresas. El $70 \%$ de los empleos en Bogotá son originados por pymes" (Canillo, 2011.)

La importancia de este proyecto de investigación, está en dar fortalecimiento al sector servicios en la búsqueda de información útil, para los bares operados, y aquellos que deseen incursionar en el sector económico. Este se denomina, según el código industrial internacional uniforme (CIIU), como: "Expendio de bebidas alcohólicas en establecimientos cerrados", investigados desde y hacia la localidad de Chapinero, en la ciudad de Bogotá. Su origen está en la capacidad para el crecimiento, la innovación y la competitividad. Algunos de estos son los factores que hacen, del proyecto de investigación, susceptible de mejora mediante conceptos y herramientas aprendidas, durante la academia formativa.

\section{MÉTODO}

La metodología se desarrolló, desde un enfoque descriptivo-explicativo, aplicado al sector expendio de bebidas alcohólicas en establecimientos cerrados.

El objetivo planteado para este proyecto es el diseñar un plan estratégico para las pequeñas y medianas empresas del sector, en la ciudad de Bogotá, con el fin de incrementar su competitividad y perdurabilidad en el tiempo.

Dentro de la metodología utilizada, se abarcaron herramientas como la segmentación y descripción del mercado, análisis DOFA y Marketing Mix. También se incluye un estudio de métodos y diseño de la cadena de suministro entre otros. Todo lo anterior, dando un gran aporte al plan estratégico planteado.

La investigación se enfoca en el diseño de un marco estratégico funcional para las pequeñas y medianas empresas, en búsqueda de perdurabilidad y competitividad. Desde otro punto de vista, se pretende disminuir el empirismo con el que las organizaciones están siendo creadas, enfocándose en el diseño de un plan estratégico. Se espera presente a grandes rasgos, particularidades como lo legal, lo técnico y lo administrativo, que los bares y/o establecimientos líderes y estructurados dejan como herencia a los demás establecimientos.

Las limitaciones que puede tener este proyecto se vieron reflejadas en la obtención de información verídica y medible, el principal problema de la investigación. La improvisación limita no sólo el correcto funcionamiento de un negocio, sino también la obtención de datos útiles, tanto para éste, como para las investigaciones que de él se realicen.

\section{RESULTADOS}

Se dio inicio con un diagnóstico integral del sector económico estudiado. Durante la realización de este diagnóstico se encontraron grandes hallazgos, los cuales dieron pautas para encaminar la investigación. En primera instancia se destacan las entidades que intervienen en el sector económico investigado este sector: 
- Alcaldia de Chapinero: Gestora de proyectos dentro de la localidad, gestiona algunos permisos y normas de los establecimientos comerciales.

- ASOBARES: Asociación de bares de Colombia; mediante esta asociación los establecimientos pueden acceder a diferentes beneficios, como es el caso del acompañamiento jurídico.
- FOPAE: Fondo de Prevención y Atención de Emergencias.

- SAYCO y ACIMPRO: Entidad encargada de la administración de recaudos realizados por derechos de autor.

A manera de información preliminar se destacan las leyes y normas que afectan el sector económico estudiado:

\section{Cuadro 1. Normatividad de los bares}

\begin{tabular}{|l|l|l|}
\hline \multicolumn{1}{|c|}{ Norma } & \multicolumn{1}{|c|}{ Marco } & \multicolumn{1}{|c|}{ Alcance } \\
\hline Ley 232 de 1995 & $\begin{array}{l}\text { Normas y requisitos para el funcionamiento de los estableci- } \\
\text { mientos comerciales. }\end{array}$ & Nivel nacional. \\
\hline Decreto 190 de 2004 & $\begin{array}{l}\text { Disposiciones del Plan de Ordenamiento Territorial (POT), uso } \\
\text { de suelos, zonas de la ciudad, calles y zonas de expansión } \\
\text { urbana. }\end{array}$ & Nivel distrital. \\
\hline Resolución 0627 de 2006 & $\begin{array}{l}\text { Establece la norma nacional de emisión de ruido y ruido am- } \\
\text { biental. }\end{array}$ & Nivel nacional. \\
\hline Articulo 2 ley de 1995 & $\begin{array}{l}\text { Comunicación de apertura de establecimiento comercial en la } \\
\text { ciudad de Bogotá. }\end{array}$ & Nivel distrital. \\
\hline Decreto 345 de 2002 & $\begin{array}{l}\text { Horarios establecidos para la realización de mi actividad co- } \\
\text { mercial en especial si corresponde a venta y/o consumo de } \\
\text { bebidas alcohólicas. }\end{array}$ & Nivel distrital. \\
\hline Ley 23 de 1982 & $\begin{array}{l}\text { Aporte correspondiente a ejecución de obras musicales dentro } \\
\text { del establecimiento por derechos de autor. }\end{array}$ & Nivel nacional. \\
\hline Decreto 675 de 1980 & $\begin{array}{l}\text { Concepto favorable del cuerpo de bomberos de la localidad de } \\
\text { Chapinero }\end{array}$ & Nivel distrital \\
\hline Acuerdo 546 de 2013 & Sistema de prevención y atención de emergencias. & Nivel distrital \\
\hline ley 1335 de 2009 & Espacios cerrados libres de humo de tabaco y cumplimiento & Nivel nacional \\
\hline Ley 9 de 1979 & $\begin{array}{l}\text { Condiciones y conceptos sanitarios favorables, vertimientos, } \\
\text { espacio público, vayas y avisos, áreas en general del estable- } \\
\text { cimiento. }\end{array}$ & Nivel nacional \\
\hline
\end{tabular}

Fuente: El autor

Tabla 1. Consolidado lista de chequeo

\begin{tabular}{|c|c|c|c|c|}
\hline Siempre & Continuamente & Ocasionalmente & Nunca & Total \\
\hline 34 & 113 & 289 & 159 & 595 \\
\hline $5 \%$ & $19 \%$ & $49 \%$ & $27 \%$ & $100 \%$ \\
\hline
\end{tabular}

Fuente. El autor 
Se obtuvieron los siguientes resultados en el diagnóstico, gracias a la realización de una lista de chequeo efectuada desde un total de 35 bares. De estos, fue analizada la frecuencia de ocurrencias de los principales obstáculos del sector, como el poco cumplimiento de la normatividad técnica y los problemas organizacionales. El consolidado presenta una fuerte tendencia $(49 \%)$ a problemáticas ocasionales asociadas, tal como se aprecia en la siguiente tabla 1.

Se desarrollaron diferentes estudios, una vez se conocieron los mayores inconvenientes de los establecimientos. El primer estudio efectuado se enfocó en el mercado. Para éste, se aplicó la herramienta de Marketing Mix, donde se analiza a profundidad la demanda, la oferta, la comercialización y la distribución.

Para el análisis de la demanda se destacan los siguientes hallazgos:

- Los gustos y preferencias de las personas son tan diversas como cambiantes a través del tiempo. No obstante, se llegan a evidenciar en cada etapa, una generalidad que es acogida por una mayoría. Dentro del estudio se evidencian esas posibles generalidades en varios aspectos, los cuales, se resaltaron dentro de la encuesta.

- En el caso del consumo de bebidas alcohólicas, se encontró en la mayoría de personas que contestaron la encuesta, su preferencia por la cerveza. También combinaban su respuesta con otra opción de licor y demostraban interés en los aperitivos y cocteles, como bebida opcional.

- Otro gran hallazgo que se evidenció, fueron las motivaciones para asistir a un bar. Cada producto busca satisfacer una necesidad; en el caso de los establecimientos predominan la diversión, la sociabilidad y el esparcimiento.

- Las personas se sienten desmotivadas para ingresar a un bar cuando está vacío o con poca clientela, así como cuando este presenta problemas de seguridad en su interior.

Los principales hallazgos encontrados en el análisis de la oferta, fueron los siguientes:

- Son pocos los establecimientos que tienen un plan estratégico completo y otros establecimientos lo confunden con el cumplimiento de normas.

- Algunos establecimientos fueron celosos con la información que dieron y los hallazgos son estrictamente visuales. Los establecimientos no acceden fácilmente a dar información por miedo al destino de sus respuestas.

- Para el marco normativo, las respuestas más consecuentes se presentaron en los establecimientos de mayor acopio. De igual manera las personas que contestaron no conocer del marco normativo, destacan que el establecimiento dio apertura de forma empírica.

- Dentro de los licores más consumidos por los clientes se destacan la cerveza y el aguardiente. Sin embargo, afirman algunos bares, el interés por el consumo de bebidas alternativas como cocteles.

- El servicio, el género musical y los precios son los aspectos más mencionados frente al valor agregado de cada establecimiento. Algunos establecimientos ofrecen más que un género musical, diferentes ambientes dentro de un mismo establecimiento. Otros establecimientos ofrecen conciencia social en vez de precios.

Para el análisis de la promoción, se destacan los componentes visualizados en la siguiente ilustración:

\section{Estructura de respuesta}

Los establecimientos organizados se ven interesados por el bienestar del cliente. Los rasgos más evidentes son las preguntas hechas 


\section{llustración 1. Análisis de la promoción}

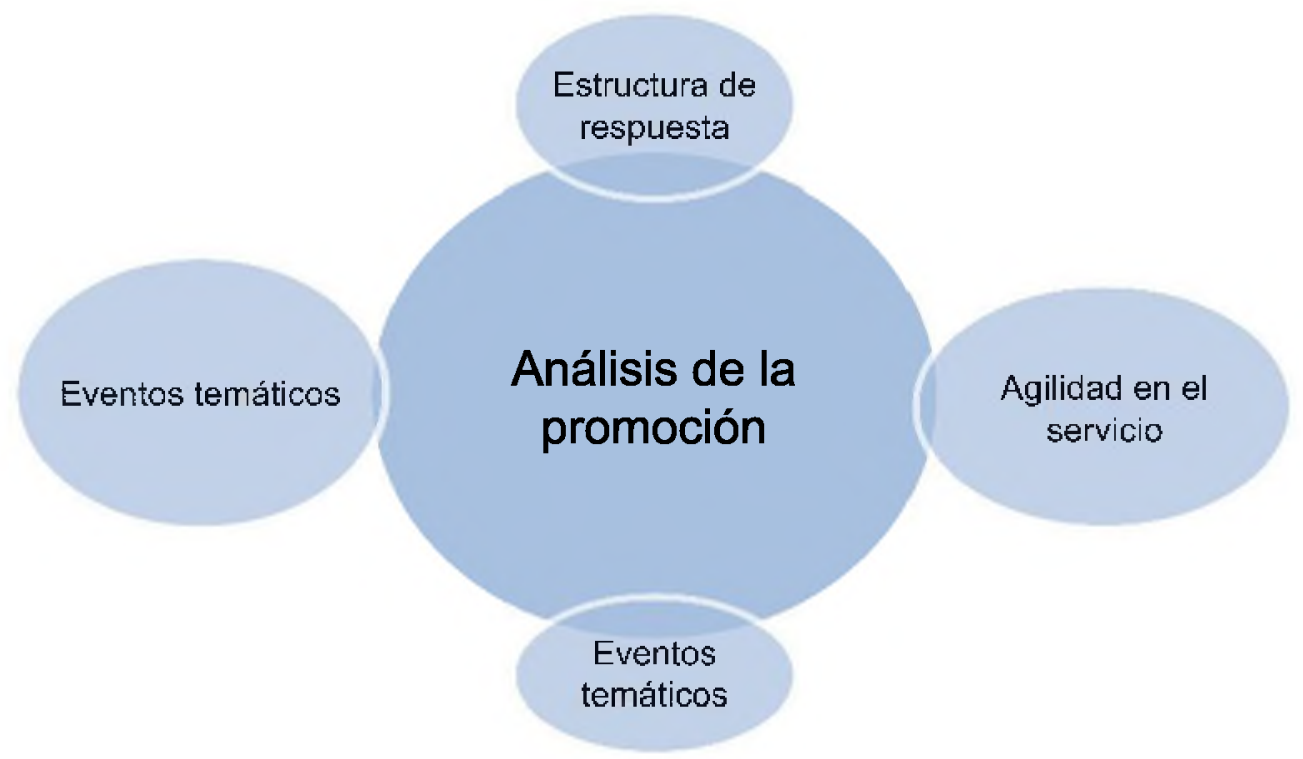

Fuente. El Autor

por los empleados sobre la calidad del servicio, la comodidad del mismo, y aquellas preguntas que indagan sus deseos. Se organizan los posibles inconvenientes con los clientes y, dentro de un marco apoyado por los empleados y socios, se crean mejores respuestas.

- Agilidad en el servicio: La mayoría de los establecimientos tienen horarios en los cuales se intensifican las ventas. Un equipo organizado y capacitado ofrece mejores rendimientos frente a la agilidad del servicio en estos horarios. Esto es evidenciable en los bares con mayor afluencia de personas. La contratación de personal llega a verse aumentada por creer que la existente, en muchos casos, no da abasto. En muchos casos solo hace falta ver la ocupación de algunos departamentos de la organización. La falta de concentración en el puesto de trabajo y el cansancio acumulado para dar mejor administración y capacitación al personal, podría llegar a verse afectado positivamente en los costos de contratación y a su vez en la calidad del servicio.
- Descuentos y promociones: Durante el transcurrir de las operaciones de un bar, el volumen de ventas se ve afectado en algunos casos por determinada hora del día. Los bares en búsqueda de incrementar las ventas en estas horas, utilizan descuentos en los cuales el precio del licor y la entrada son los principales afectados. El bar utiliza este instrumento para poder dar mayor afluencia de personas que se vean motivadas a ingresar al establecimiento en vista de los descuentos. De esta manera los clientes verán de manera positiva la existencia de un buen servicio. Las personas inconscientemente tienden a rechazar tanto un restaurante como un bar vacio. Esta clase de descuentos dentro de un lapso de tiempo, normalmente se les denomina "Happy Hour", haciendo referencia a la felicidad experimentada en esta hora, en vista de los bajos precios. Esta estrategia consiste en ofrecer dos tragos por el precio de uno durante un periodo de tiempo determinado.

- Eventos temáticos: Algunos establecimientos dan uso a sus locaciones para lanzar, efectuar e impulsar trabajos alternos al bar. Estos bares destinan su espacio en algunos días 
del inicio de semana, para mostrar trabajos, danzas, cortometrajes y foros entre otras actividades no propias de un bar, más sí de su mercado objetivo.

- Para el análisis de la distribución se destacan los agentes involucrados en la cadena de suministro, como se ve en el siguiente esquema 1.

El análisis de la distribución destaca, de manera general, la importancia de desarrollar las actividades de logistica interna (almacenamiento de insumos), logistica externa (inventarios y escogencia de proveedores), actividades operativas (actividades propias del establecimiento), actividades de marketing (impulso de marca) y servicios post-venta (quejas y reclamos), junto con los agentes internos y externos. Para finalizar el estudio de mercado, se realizó un análisis DOFA (Debilidades, Oportunidades, Fortalezas y Amenazas), teniendo en cuenta las principales estrategias utilizadas en los bares encuestados. A continuación el consolidado del análisis DOFA:

\section{Esquema 1. Agentes cadena de Valor}

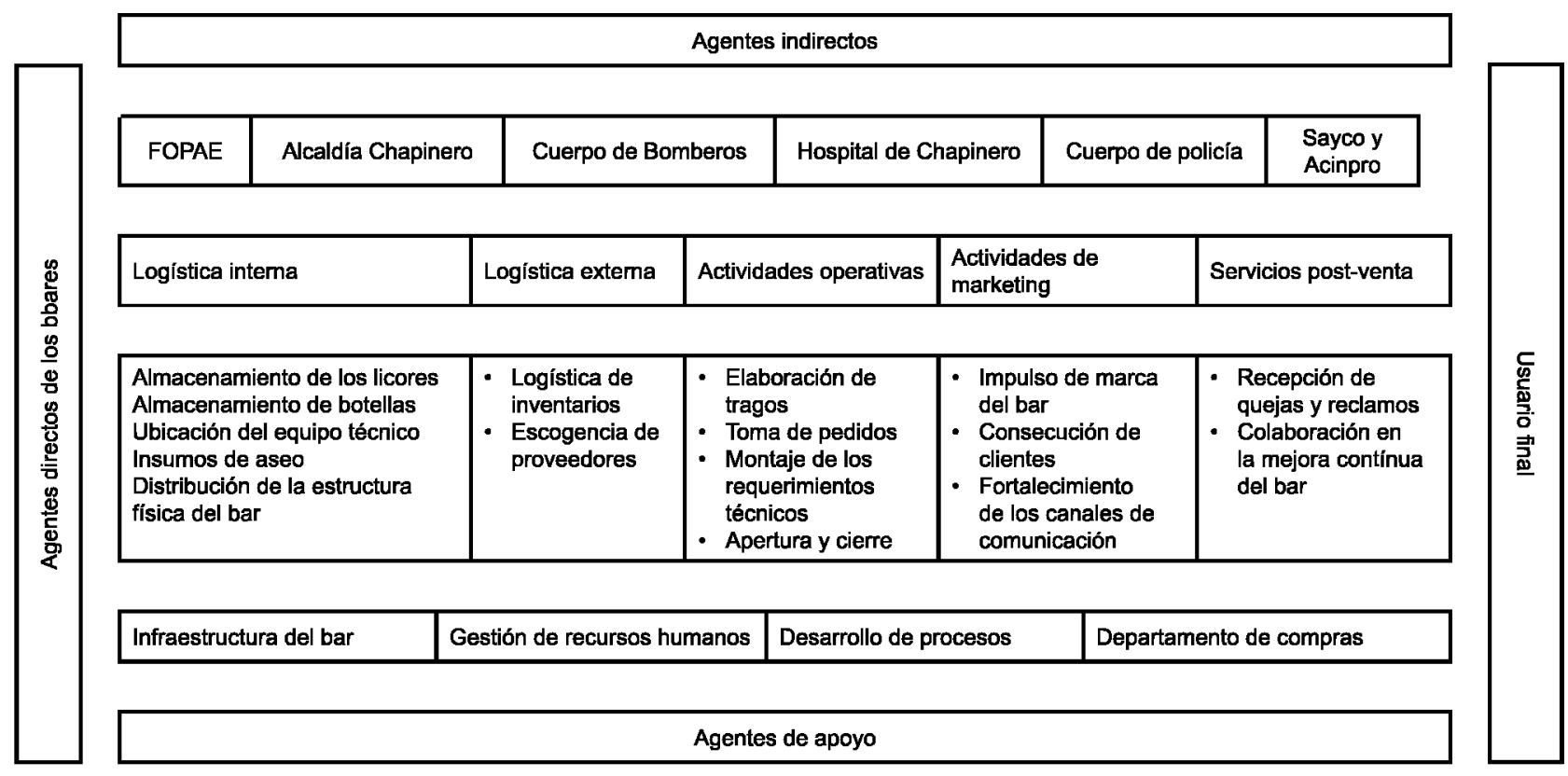

Fuente. El autor

Las estrategias expuestas en el anterior esquema, sintetizan el esfuerzo de algunos establecimientos por dar competitividad y perdurabilidad a su negocio. Estas estrategias presentan la particularidad de funcionar, si se contemplan en la prevención de inconvenientes dentro del bar. En los resultados presentados del estudio técnico, se destaca un análisis de la macro y micro-localización, junto a los requerimientos de seguridad exigidos por las entidades intervinientes del sector económico.
En la macro-localización se destacan las características propias de la localidad, proyectos y beneficios que trae la misma, para el sector económico investigado. La microlocalización concentra sus esfuerzos en la adecuación técnica del establecimiento. Para dicho objetivo se muestra, a continuación, un esquema con las características básicas de un local, y la importancia de la correcta selección del mismo. 
Los factores a tener en cuenta dentro de la matriz a continuación, actúan como filtros realizados por datos generales, características interiores, características exteriores y características del sector.

Los datos generales están compuestos por estrato, área, baños y antigüedad. Las características del interior están compuestas por iluminación, servicios públicos y salidas de emergencia. Las características del exterior están compuestas por parqueaderos, zona de recepción, acceso al establecimiento y vigilancia del exterior. Las características del sector son: las vías de acceso al bar en buen estado, ubicación sobre una vía principal, transporte público cercano y zona comercial. La matriz busca dar claridad y objetividad respecto a la escogencia de un local dónde efectuar la actividad económica, y la asignación de posibles locaciones, para posteriormente darles una calificación, de acuerdo a los parámetros establecidos. La locación con mayor puntuación será la que preste mayor seguridad y comodidad a los clientes.

Frente al cumplimiento de la normatividad técnica, el Acuerdo 20 de 1995, el cual determina el código de construcción, decreta los siguientes parámetros a tener en cuenta por cualquier establecimiento comercial. El no cumplimiento de la normatividad exigida por las diferentes entidades, representa para los establecimientos un foco de riesgo para el sellamiento del local.
Así mismo, es necesario estipular los planes de emergencia y los responsables directos de la creación del mismo, tal como se muestra en el siguiente esquema con su respectiva explicación:

- El grupo de planificación tendrá a cargo la situación actual del establecimiento, la documentación y los recursos necesarios para desarrollar el plan de emergencias.

- El grupo de operaciones desarrollará los planes de acción pertinentes.

- El grupo de logística tendrá a cargo el aprovisionamiento, la distribución y los servicios requeridos en la elaboración del plan de emergencias.

- El grupo de administración y finanzas se encargará de los costos y presupuestos del plan.

- Estos esquemas deben cumplir con condiciones de visibilidad en áreas de riesgo, información de comunicación con los responsables, planos y un directorio de los organismos de control, tanto interno como externos.

- La estructura donde se realice la actividad economía debe estar debidamente señaliza de acuerdo a la normatividad exigida, tal como se muestra en la siguiente imagen suministrada por el SENA:

\section{Cuadro 2. Código de Construcción}

\begin{tabular}{l} 
Medios de evacuación: Vías libres y continuas que partiendo desde cualquier punto de una edificación \\
conducen a un lugar o una vía pública. Cada medio de evacuación consta de partes separadas y distintas: \\
salida, acceso a ésta. \\
\hline $\begin{array}{l}\text { Módulo de salida: Ancho mínimo de salida, para el cómodo movimiento de una fila de personas a lo largo } \\
\text { de un medio de evacuación; el ancho de la salida debe ser de } 1.8 \mathrm{~m} \text {. }\end{array}$ \\
\hline $\begin{array}{l}\text { Nivel de calle: Piso o nivel de piso accesible desde la calle o el exterior de la edificación, provisto de entrada } \\
\text { principal a una altura no mayor de } 7 \text { escalones sobre el nivel del suelo. }\end{array}$ \\
\hline $\begin{array}{l}\text { Rampa: Plano inclinado dispuesto para subir y bajar en un sentido determinado de circulación, el grado de } \\
\text { inclinación debe ser máximo de } 20^{\circ} \text { y debe permanecer constante durante todo el trayecto, el piso debe ser } \\
\text { antideslizante. Las rampas son necesarias cuando hay cambios en la altura del establecimiento para perso- } \\
\text { nas con discapacidad }\end{array}$ \\
\hline
\end{tabular}


Salida: Parte de un medio de evacuación, separada de los demás espacios de la edificación por construcciones o equipos como se especifica en este capítulo, y que proporciona una vía de recorrido protegida hasta la descarga de salida. Puede incluir escalera a prueba de humo, corredores, balcones exteriores, rampas y puertas.

Vía pública: Calle, callejón u otro espacio seguro, abierto al exterior para fines de uso público y con un ancho no menor de $3 \mathrm{~m}$, los punto de encuentro debe tener las siguientes características: poseer instalaciones físicas adecuadas para las personas jóvenes y adultas. Mantiene sus espacios abiertos todos los días de la semana, es de carácter amplio y no tiene edificaciones o puntos de riesgo geográfico alrededor.

Fuente. El Autor

Esquema 2. Análisis DOFA

\begin{tabular}{|c|c|c|}
\hline EXTERNO & $\begin{array}{l}\text { OPORTUNIDADES } \\
\text { - Búsqueda constante de espacios } \\
\text { de esparcimiento por parte de las } \\
\text { personas. } \\
\text { - Teoría del bar lleno. } \\
\text { - Fechas especiales. } \\
\text { - Servicios adicionales. }\end{array}$ & $\begin{array}{l}\text { AMENAZAS } \\
\text { - Gustos y preferencias cambiantes por } \\
\text { parte de los consumidores. } \\
\text { - Frecuencia de asistencia variable por } \\
\text { factores externos. } \\
\text { - Inestabilidad jurídica por parte de los } \\
\text { organismos de control. } \\
\text { - Clima y la competencia indirecta. }\end{array}$ \\
\hline $\begin{array}{l}\text { FORTALEZAS } \\
\text { Manejo libre de precios. } \\
\text { - Variedad de géneros musicales. } \\
\text { - Programas de fidelización. } \\
\text { - Facilidad en la consecución de } \\
\text { proveedores. } \\
\text { - Variedad de tragos. }\end{array}$ & $\begin{array}{l}\text { Estrategia (F-O) } \\
\text { - Búsqueda constante en la } \\
\text { ampliación de la carta de licores. } \\
\text { - Descuentos y tratos especiales } \\
\text { para clientes que festejen fechas } \\
\text { importantes en el establecimiento, } \\
\text { - Descuentos y tratos especiales a } \\
\text { las primeras personas que lleguen } \\
\text { al establecimiento. }\end{array}$ & $\begin{array}{l}\text { Estrategia (F-A) } \\
\text { - Establecimientos con espacios } \\
\text { alternativos como cine foros, } \\
\text { cuentería y humor. } \\
\text { - Ambientes musicales definidos para } \\
\text { un nicho de mercado determinado. }\end{array}$ \\
\hline $\begin{array}{l}\text { DEBILIDADES } \\
\text { - Falta de conocimiento de la } \\
\text { normatividad jurídica y legal. } \\
\text { - Falta de un plan estratégico de } \\
\text { negocios. } \\
\text { - Inestabilidad laboral y dificultades } \\
\text { en la comunicación interna. } \\
\text { - Frecuencia de asistencia variable } \\
\text { por factores internos. }\end{array}$ & $\begin{array}{l}\text { Estrategia (D-O) } \\
\text { - Formalización de los cargos, } \\
\text { capacitación y estructura jurídica } \\
\text { del establecimiento. } \\
\text { - Alistamiento oportuno del local y } \\
\text { requerimientos del evento a cubrir. } \\
\text { - Creación de un plan estratégico. }\end{array}$ & $\begin{array}{l}\text { Estrategia (D-A) } \\
\text { - Conocimiento anticipado del marco } \\
\text { normativo que rige en los bares. } \\
\text { - Organización del perfil de cargos y } \\
\text { responsabilidades. } \\
\text { - Seguimiento post-venta en búsqueda } \\
\text { de nuevas tendencias y opiniones del } \\
\text { concepto de un bar. }\end{array}$ \\
\hline
\end{tabular}

Fuente. El Autor 
LÍNEA DE INVESTIGACIÓN: EMPRENDIMIENTO Y EMPRESARIALISMO

\section{Esquema 3. Adecuación local}

\begin{tabular}{|c|c|c|c|c|c|c|c|c|c|c|c|c|c|c|c|c|}
\hline \multirow[b]{4}{*}{$\begin{array}{l}\text { Criterios de evaluación } \\
\text { - Cumple totalmente (5). } \\
\text { - Cumple en gran } \\
\text { medida.(4) } \\
\text { - Cumple en una medida } \\
\text { aceptable. (3) } \\
\text { - Casi no cumple.(2) } \\
\text { - No cumple. (1) }\end{array}$} & \multicolumn{16}{|c|}{ MATRIZ SELECCIÓN DE OPCIONES } \\
\hline & \multicolumn{16}{|c|}{ FILTROS } \\
\hline & \multicolumn{4}{|c|}{ Datos generales } & \multicolumn{3}{|c|}{\begin{tabular}{|c|} 
Caracteristicas \\
interiores
\end{tabular}} & \multicolumn{4}{|c|}{$\begin{array}{c}\text { Caracteristicas } \\
\text { exteriores }\end{array}$} & \multicolumn{4}{|c|}{$\begin{array}{c}\text { Caracteristicas } \\
\text { del sector }\end{array}$} & \\
\hline & 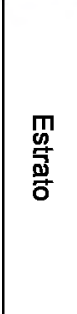 & 尝 & 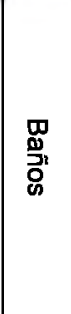 & 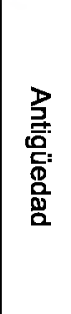 & 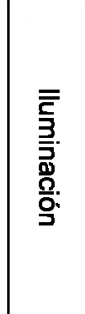 & 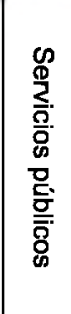 & 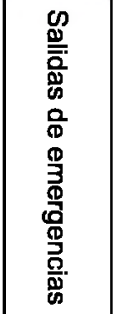 & 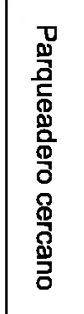 & 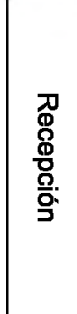 & 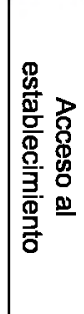 & 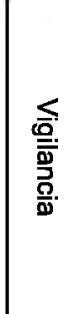 & 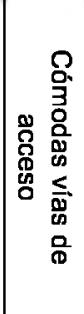 & 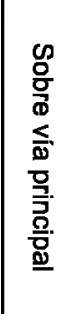 & 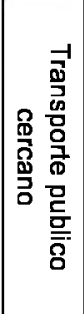 & 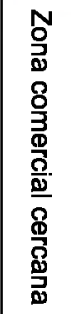 & 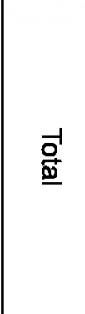 \\
\hline Ponderación & $10 \%$ & $10 \%$ & $4 \%$ & $4 \%$ & $4 \%$ & $4 \%$ & $12 \%$ & $10 \%$ & $4 \%$ & $6 \%$ & $5 \%$ & $12 \%$ & $8 \%$ & $5 \%$ & $2 \%$ & $100 \%$ \\
\hline
\end{tabular}

Fuente. El autor

\section{Esquema 4. Plan de Emergencias}

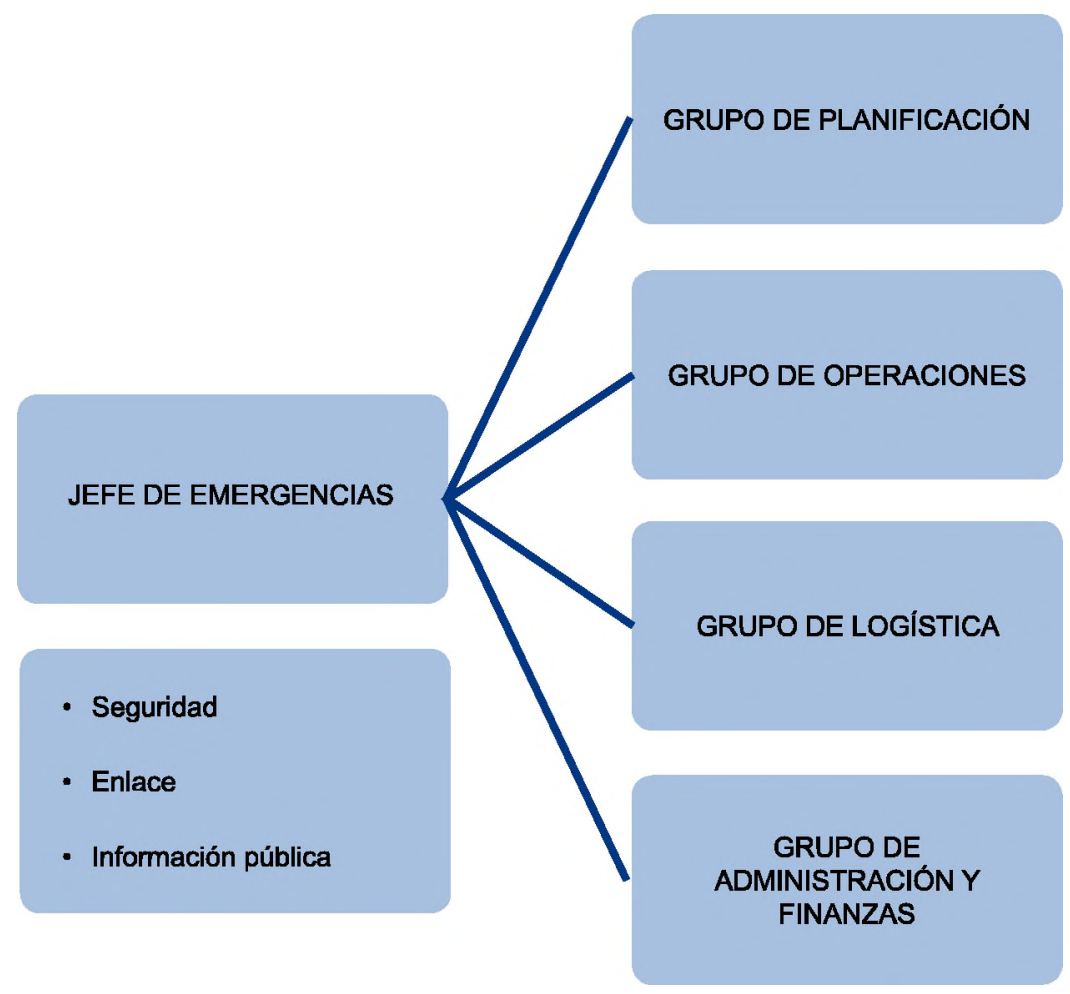

Fuente. El Autor 
llustración 2. Señalización
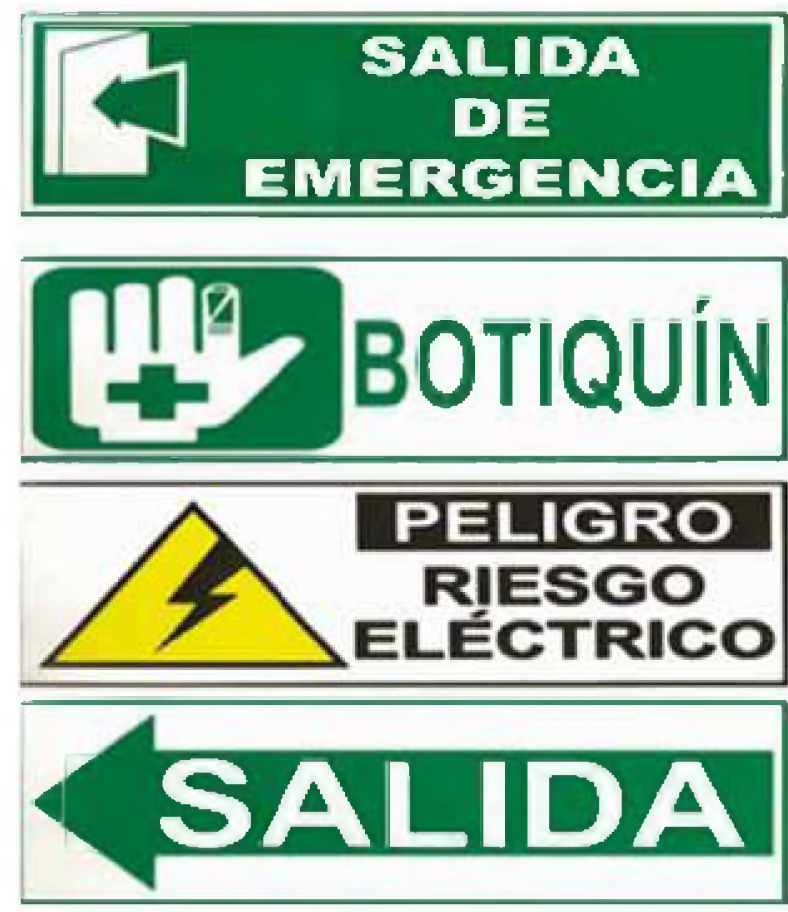

Fuente: SENICOL.

La investigación, destaca también dentro del estudio de métodos, los cargos departamentalizados, dentro de un establecimiento, tal como se muestra en los siguientes esquemas, donde se destacan los cargos con su respectiva labor:

La investigación, destaca un flujo de procesos general, el cual sirve de parámetro inicial para el plan estratégico, aplicado a cualquier bar que desee implementar el plan estratégico.

El resultado del estudio administrativo contempla la creación de un organigrama y el flujo de procesos correspondientes a la selección de personal, parte fundamental en el correcto funcionamiento de un bar. A continuación se muestra un organigrama general distribuido por departamentos, junto con las pautas para la correcta selección.

El organigrama diferencia los departamentos correspondientes a la barra, el sonido, el servicio a la mesa, la seguridad del estableci-
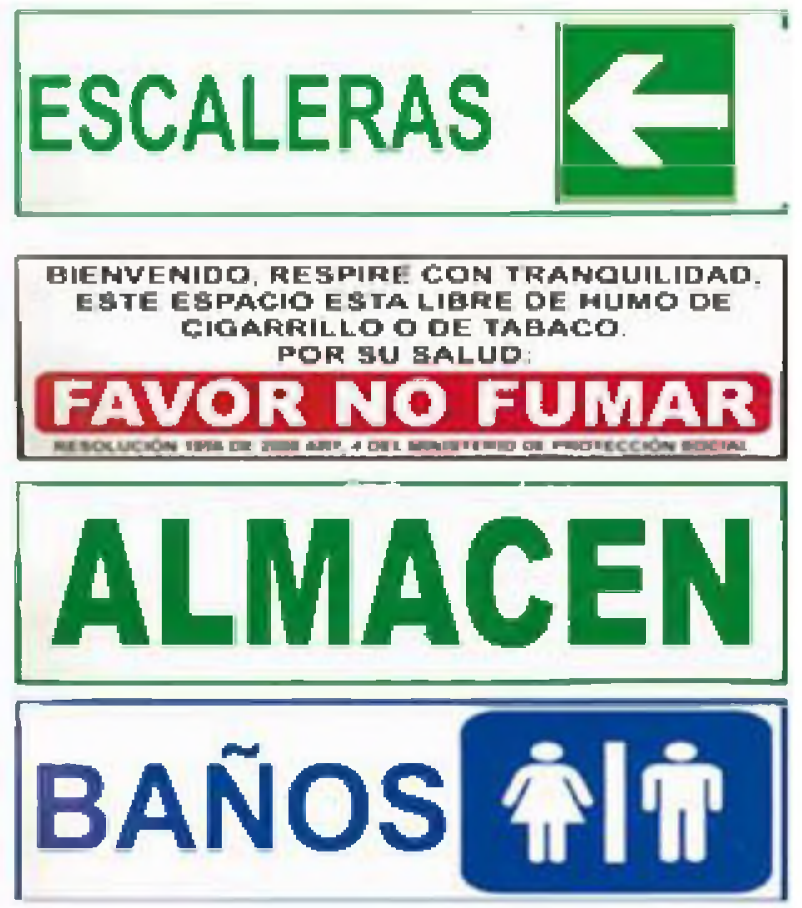

miento y los servicios varios. Cada uno de los puestos en este organigrama referenciado, deben ser ocupados por personal capacitado. Ésta selección tiene la naturaleza de llevar el siguiente flujo. Dentro de las técnicas de selección, se encuentran los siguientes niveles de medición:

- Entrevista de selección dirigida y libre: la entrevista puede ser estructurada y estandarizada. Esta determina la capacidad básica de cada candidato para cubrir determinado cargo dentro del bar.

- Conocimientos o capacidades generales y específicas: busca medir el grado de conocimientos profesionales o técnicos, como coctelería, operatividad de consolas y sonido. Por otro lado, se busca medir las habilidades para determinadas tareas como la caja registradora, comunicación y servicio.

- Pruebas psicológicas: se utilizan como medida del desempeño. Se basan en muestras 
estadísticas para la comparación y se compaginan en condiciones estandarizadas; necesitan de un pronóstico, de tener validez y poseer precisión. Es válido para cargos con alta responsabilidad del establecimiento como la administración.

- Pruebas de personalidad: busca la integración única de características medibles que se relacionan con aspectos permanentes $y$ consistentes de una persona. Ejemplo: extrovertido, agradables, consciente, neurótico y abierto a las experiencias.

- Técnicas de simulación: se concentran en el trato grupal y de acción social. Se utilizan como complemento de las pruebas anteriores, ya que al hacer una dramatización de los acontecimientos de la tarea en el bar se puede ver con claridad las reacciones del aspirante.

\begin{tabular}{|l|}
\multicolumn{1}{c|}{ Jefe de barra } \\
\hline - Alistamiento del inventario \\
- Preparación del pedido \\
- Alistamiento de la caja \\
- Recepción de dinero por parte del bartender y meseros \\
\hline
\end{tabular}

\begin{tabular}{|l|}
\hline \multicolumn{1}{|c|}{ Bartender } \\
\hline - Alistamiento de la barra \\
- Alistamiento de la carta de tragos \\
- Preparación de bebidas alcohólicas pedidas por los clientes \\
y cuerpo de meseros \\
- Entrega del dinero al jefe de barra \\
- Cierre de la barra
\end{tabular}

\begin{tabular}{|l|}
\hline \multicolumn{1}{|c|}{ Ingeniero de sonido } \\
\hline - Preparación de la cabida de sonido \\
- Alistamiento de la consola maestra \\
- Configuración de la consola maestra \\
- Orientar los requerimientos al Roadie \\
y operador de sonido \\
- Cierre de equipos y de consola \\
maestra \\
\hline
\end{tabular}

\begin{tabular}{|l|}
\hline \multicolumn{1}{|c|}{ Roadie } \\
\hline - Alistamiento e instalación de \\
cableado y equipo técnico del sonido \\
Orientar y cumplir requerimientos \\
de los grupos en la ubicación de \\
instrumentos \\
- Desistalación del cableado y equipo \\
técnico del sonido \\
\hline
\end{tabular}

\begin{tabular}{|l|}
\hline \multicolumn{1}{|c|}{ Operador de sonido } \\
\hline - Alistamiento de la consola del sonido \\
- Reproducción de la música durante \\
el evento \\
- Desinstalación de I aconsola de \\
sonido
\end{tabular}

\begin{tabular}{|l|}
\multicolumn{1}{c|}{ Capitán de meseros } \\
\hline - Alistar el equipo de meseros, indicando las características \\
del evento \\
- Atneder solicitudes de los clientes \\
- Realizar sugerencias de la carta de licores \\
- Tomar pedido de clientes \\
- Llevar pedido a la caja \\
- Llevar el pedido a la mesa \\
- Recibir el dinero de los clientes \\
- Administrar las propinas a los meseros \\
\hline
\end{tabular}

\begin{tabular}{|l|}
\multicolumn{1}{c|}{ Meseros } \\
\hline - Atender solicitudes de los clientes \\
- Realizar sugerencias de la carta de licores \\
- Tomar pedido de clientes \\
- Llevar pedido a la caja \\
- Llevar el pedido a la mesa \\
- Recibir el dinero de los clientes \\
\hline
\end{tabular}

\section{Seguridad externa}

- Realizar el alistamiento de la puerta

- Extender la invitación a los posibles clientes

- Realizar la requisa de manera amable y ética a los clientes

- Velar por la salida ordenada del establecimiento

\section{Seguridad interna}

- Velar por la seguridad de los clientes una vez entren al establecimiento

- Velar por la salida ordenada del establecimiento

Fuente. El Autor 


\section{Esquema 5. Flujo de procesos de un Bar}
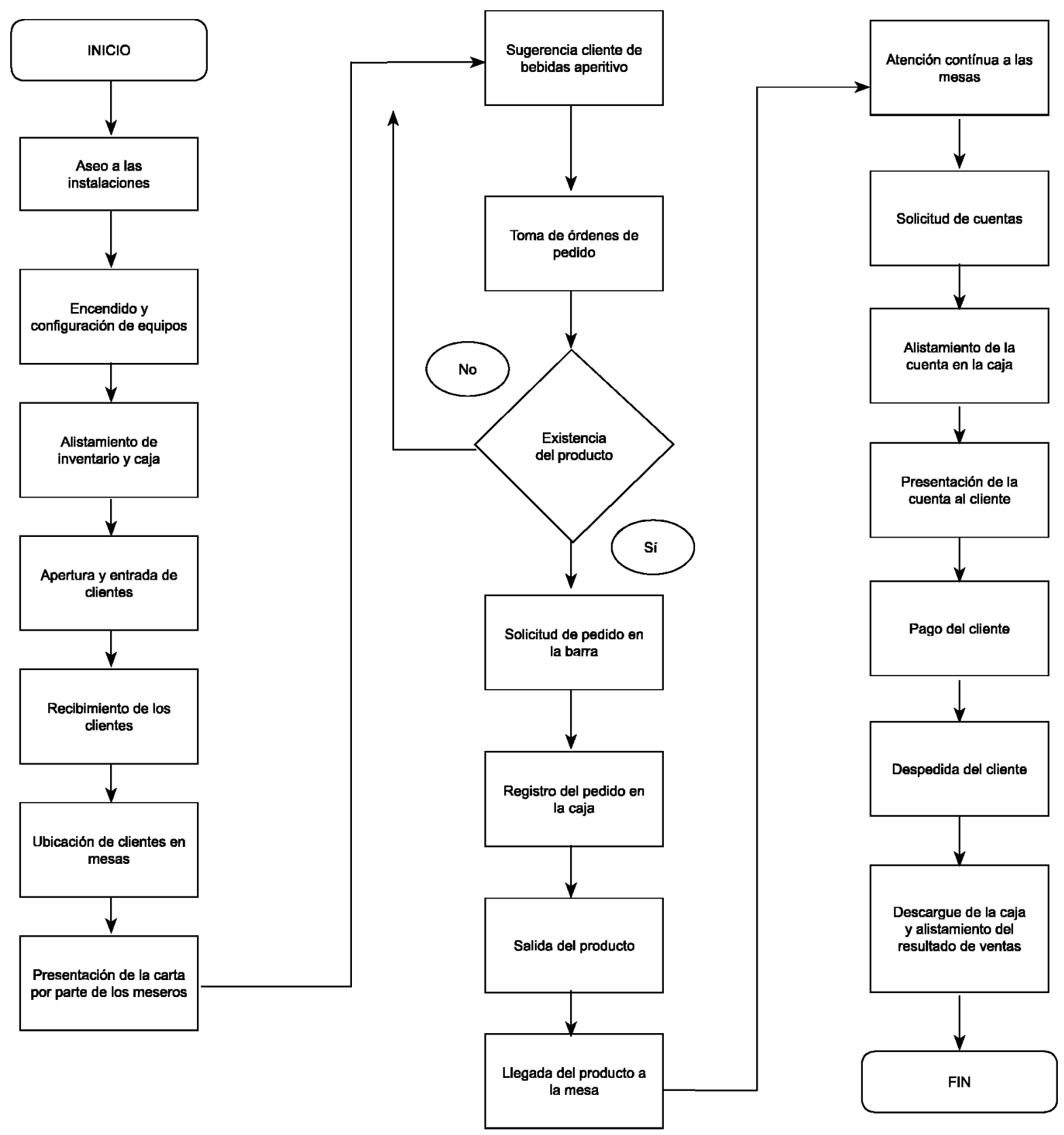

Fuente. El Autor

Las anteriores técnicas de selección hacen parte de la contratación laboral efectiva. Ésta requiere del análisis de diferentes características, las cuales, se exponen mejor en una contratación por competencias laborales generales.
Para concluir con los resultados d el estudio, se muestra el plan estratégico tomado de cada hallazgo, junto con el objetivo, la meta, la estrategia, las actividades a realizar y el resultado esperado. 


\section{Esquema 6. Organigrama Bar}

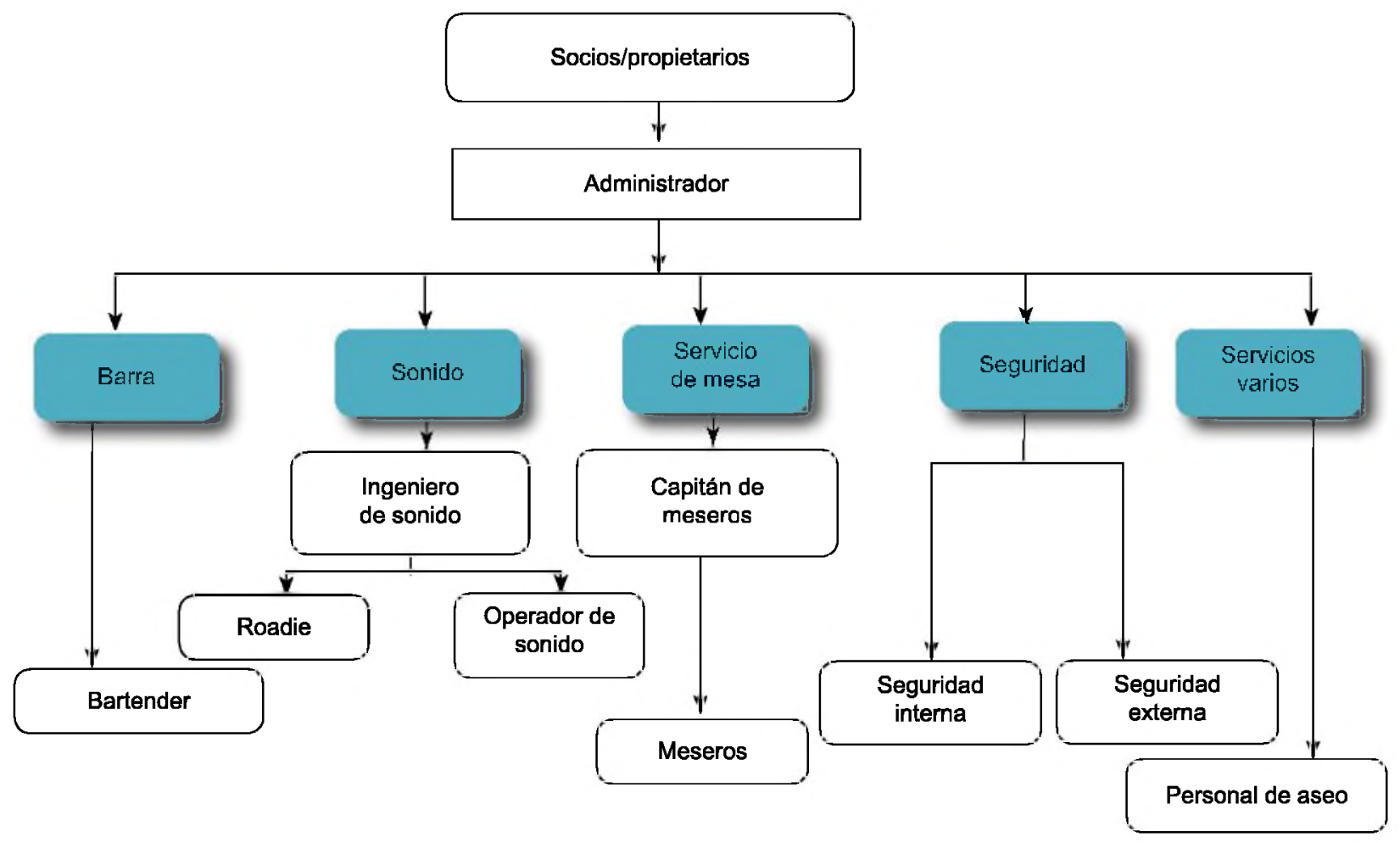

Fuente. El Autor

\section{Esquema 7. Flujo selección de personal}

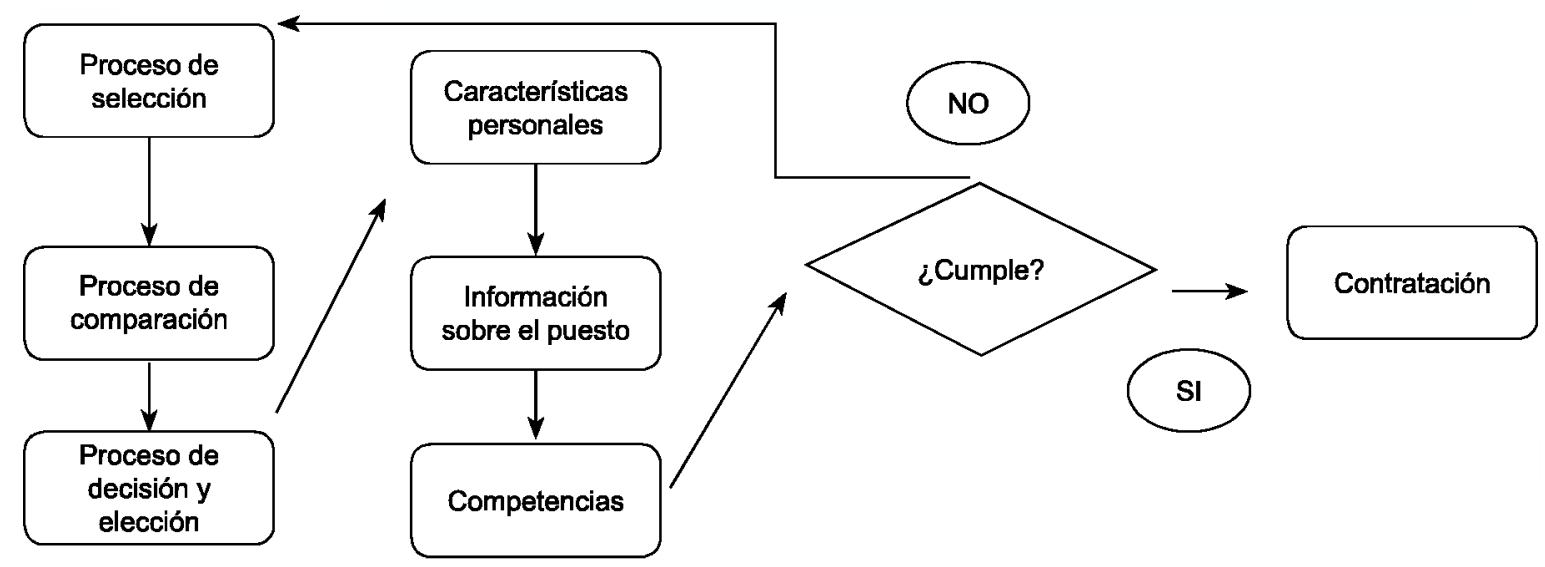

Fuente. El Autor 


\section{Esquema 8. Plan estratégico}

\begin{tabular}{|c|c|c|c|c|}
\hline Objetivo & Mata & Estrategla & Actividad & Resultado \\
\hline $\begin{array}{l}\text { Diseñar un estudio } \\
\text { para la correcta } \\
\text { selección del } \\
\text { mercado. }\end{array}$ & $\begin{array}{l}\text { El establecimiento } \\
\text { enfoca sus } \\
\text { acciones en el } \\
\text { segmento indicado. }\end{array}$ & $\begin{array}{l}\text { Realizar un } \\
\text { análisis de la } \\
\text { demanda }\end{array}$ & $\begin{array}{l}\text { - Definir parámetro iniciales del mercado. } \\
\text { - Segmentación del mercado de forma } \\
\text { - Demográfica, pictográfica y conductual. } \\
\text { - Realir muestra representativa. } \\
\text { - Aplicar encuesta. } \\
\end{array}$ & $\begin{array}{l}\text { - Hallazgos } \\
\text { importantes para } \\
\text { el establecimiento } \\
\text { en el ámbito de la } \\
\text { demanda. }\end{array}$ \\
\hline $\begin{array}{l}\text { Diseñar un estudio } \\
\text { para analizar la } \\
\text { competencia. }\end{array}$ & \begin{tabular}{|l|} 
El establecimiento, \\
conoce las \\
caracteristicas de \\
la competencia \\
generando un valor \\
agregado.
\end{tabular} & $\begin{array}{l}\text { Realizar un } \\
\text { análisis de la } \\
\text { oferta }\end{array}$ & $\begin{array}{l}\text { - Definir parámetro iniciales de los } \\
\text { competidores. } \\
\text { - Realizar un contacto a los competidores } \\
\text { directos mediante una encuesta. } \\
\text { - Analizar las características de la } \\
\text { competencia }\end{array}$ & $\begin{array}{l}\text { - Hallazgos } \\
\text { importantes para } \\
\text { definir el precio del } \\
\text { servicio. } \\
\text { - Conocimiento del } \\
\text { valor agregado de la } \\
\text { competencia. }\end{array}$ \\
\hline $\begin{array}{l}\text { Diseñar un estudio } \\
\text { para conocer } \\
\text { las estrategias } \\
\text { para acercar el } \\
\text { establecimiento al } \\
\text { cliente. }\end{array}$ & \begin{tabular}{|l|} 
El establecimiento \\
conoce los medios \\
de comunicación \\
efectiva frente a los \\
clientes.
\end{tabular} & $\begin{array}{l}\text { Realizar un } \\
\text { análisis de la } \\
\text { comercialización }\end{array}$ & $\begin{array}{l}\text { - Descripción de la comercialización } \\
\text { - Definir canales de comercialización más } \\
\text { utilizados por el sector económico. } \\
\text { - Crear canales de publicidad y promoción. } \\
\text { - Uso de redes sociales. }\end{array}$ & $\begin{array}{l}\text { - El establecimiento } \\
\text { aumenta su número } \\
\text { de seguidores y } \\
\text { clientes potenciales. }\end{array}$ \\
\hline $\begin{array}{l}\text { Diseñar un estudio } \\
\text { para determinar } \\
\text { la percepción de } \\
\text { un cliente frente al } \\
\text { servicio. }\end{array}$ & \begin{tabular}{|l|} 
El establecimiento \\
conoce la opinión \\
acerca del servicio \\
ofrecido, mejorando \\
las problemáticas \\
reflejadas por los \\
clientes. \\
\end{tabular} & $\begin{array}{l}\text { Realizar un } \\
\text { análisis de las } \\
\text { personas }\end{array}$ & $\begin{array}{l}\text { - Captura de datos de los clientes. } \\
\text { - Pregunta directa. } \\
\text { - Seguimiento por correo electrónico. } \\
\text { - Redes sociales. } \\
\text { - Indicadores de satisfacción de clientes. }\end{array}$ & $\begin{array}{l}\text { - El establecimiento } \\
\text { conoce las } \\
\text { problemáticas } \\
\text { presentadas por los } \\
\text { clientes frecuentes, } \\
\text { generando así la } \\
\text { mejora continua. } \\
\end{array}$ \\
\hline $\begin{array}{l}\text { Diseñar un estudio } \\
\text { para determinar } \\
\text { las características } \\
\text { tanto internas } \\
\text { como externas del } \\
\text { establecimiento }\end{array}$ & \begin{tabular}{|l|} 
El establecimiento \\
conoce sus \\
debilidades, \\
fortalezas, \\
amenazas y \\
oportunidades y \\
genera estrategias \\
para atacar las \\
problemáticas
\end{tabular} & $\begin{array}{l}\text { Realizar un } \\
\text { análisis DOFA }\end{array}$ & $\begin{array}{l}\text { - Análisis de las fortalezas. } \\
\text { - Análisis de las oportunidades. } \\
\text { - Análisis de las debilidades. } \\
\text { - Análisis de las amenazas. } \\
\text { - Plan de marketing. }\end{array}$ & $\begin{array}{l}\text { - Genera estrategias } \\
\text { para mejorar el } \\
\text { servicio y hacer el } \\
\text { establecimiento más } \\
\text { eficiente. } \\
\text { - Se crea un plan de } \\
\text { marketing. }\end{array}$ \\
\hline $\begin{array}{l}\text { Definir la ubicación } \\
\text { y zona donde ubicar } \\
\text { mi establecimiento. }\end{array}$ & $\begin{array}{l}\text { El establecimiento } \\
\text { conoce las } \\
\text { caracteristicas del } \\
\text { sector, la localidad } \\
\text { y barrio donde va a } \\
\text { ubicarse }\end{array}$ & \begin{tabular}{|l|} 
Realizar un \\
análisis de \\
la marco y \\
microlocalización
\end{tabular} & $\begin{array}{l}\text { - Realizar un acercamiento a las } \\
\text { características de la macro y } \\
\text { microlocalización. } \\
\text { - Documentar el establecimiento frente a } \\
\text { los requerimientos mínimos físicos para } \\
\text { establecer un negocio. } \\
\text { - Realizar una matriz de locación teniendo } \\
\text { en cuenta los requisitos exigidos. }\end{array}$ & \begin{tabular}{|l|} 
El establecimiento \\
conoce las \\
características del \\
sector donde está \\
ubicado. \\
- El establecimiento \\
conoce de la \\
normatividad técnica \\
exigida. \\
\end{tabular} \\
\hline $\begin{array}{l}\text { Definir los } \\
\text { requerimientos } \\
\text { frente a las } \\
\text { necesidades del } \\
\text { establecimiento. }\end{array}$ & $\begin{array}{l}\text { El establecimiento } \\
\text { dispone de todo } \\
\text { el equipo físico } \\
\text { necesario para } \\
\text { funcionar de } \\
\text { manera adecuada. } \\
\end{array}$ & $\begin{array}{l}\text { Realizar } \\
\text { un análisis } \\
\text { del equipo, } \\
\text { maquinaria } \\
\text { tecnología de } \\
\text { un bar } \\
\end{array}$ & $\begin{array}{l}\text { - Realizar un análisis de los requerimientos } \\
\text { necesarios para funcionar. } \\
\text { - Analizar la tecnología. } \\
\text { - Analizar los equipos. }\end{array}$ & $\begin{array}{l}\text { El establecimiento } \\
\text { dispone de los } \\
\text { requerimientos } \\
\text { necesarios para } \\
\text { funcionar. }\end{array}$ \\
\hline $\begin{array}{l}\text { Definir los } \\
\text { requerimientos } \\
\text { frente a las } \\
\text { necesidades del } \\
\text { establecimiento. }\end{array}$ & $\begin{array}{l}\text { El establecimiento } \\
\text { dispone de todo } \\
\text { el equipo físico } \\
\text { necesario para } \\
\text { funcionar de } \\
\text { manera adecuada. }\end{array}$ & \begin{tabular}{|l} 
Realizar \\
un análisis \\
del equipo, \\
maquinaria \\
tecnología de \\
un bar \\
\end{tabular} & $\begin{array}{l}\text { - Realizar un análisis de los requerimientos } \\
\text { necesarios para funcionar. } \\
\text { - Analizar la tecnología. } \\
\text { - Analizar los equipos. }\end{array}$ & $\begin{array}{l}\text { El establecimiento } \\
\text { dispone de los } \\
\text { requerimientos } \\
\text { necesarios para } \\
\text { funcionar. }\end{array}$ \\
\hline
\end{tabular}


LÍNEA DE INVESTIGACIÓN: EMPRENDIMIENTO Y EMPRESARIALISMO

\begin{tabular}{|c|c|c|c|c|}
\hline Objetivo & Meta & Estrategla & Actividad & Resultado \\
\hline $\begin{array}{l}\text { Definir las } \\
\text { condiciones ideales } \\
\text { de funcionamiento. }\end{array}$ & $\begin{array}{l}\text { El establecimiento } \\
\text { cumple con todos } \\
\text { los requerimientos } \\
\text { físicos mínimos } \\
\text { exigidos. }\end{array}$ & $\begin{array}{l}\text { Realizar un } \\
\text { análisis de la } \\
\text { normatividad } \\
\text { exigida en } \\
\text { el sector } \\
\text { económico, }\end{array}$ & $\begin{array}{l}\text { - Documentar los requerimientos mínimos } \\
\text { exigidos. } \\
\text { - Generar un plan de evacuación y } \\
\text { contingencias. } \\
\text { - Acondicionar el establecimiento de } \\
\text { acuerdo a las exigencias. }\end{array}$ & $\begin{array}{l}\text { El establecimiento } \\
\text { cumple con la } \\
\text { normatividad exigida. }\end{array}$ \\
\hline $\begin{array}{l}\text { Definir los métodos } \\
\text { de operatividad } \\
\text { y funcionamiento } \\
\text { dentro del } \\
\text { establecimiento. }\end{array}$ & $\begin{array}{l}\text { El establecimiento } \\
\text { conoce el } \\
\text { funcionamiento } \\
\text { interno del } \\
\text { establecimiento. }\end{array}$ & $\begin{array}{l}\text { Realizar un } \\
\text { estudio de } \\
\text { métodos }\end{array}$ & $\begin{array}{l}\text { - Departamentalizar los cargos dentro de un } \\
\text { bar. } \\
\text { - Crear la cadena de suministro. } \\
\text { - Crear la matriz de cargas de trabajo. } \\
\text { - Generar las buenas prácticas de los } \\
\text { establecimientos. } \\
\text { - Fijar un stock de inventario }\end{array}$ & $\begin{array}{l}\text { - El establecimiento } \\
\text { genera buenas } \\
\text { prácticas dentro de } \\
\text { sus instalaciones, } \\
\text { optimizando los } \\
\text { requerimientos. }\end{array}$ \\
\hline $\begin{array}{l}\text { Definir la planeación } \\
\text { estratégica. }\end{array}$ & $\begin{array}{l}\text { El establecimiento } \\
\text { crea un plan } \\
\text { estratégico } \\
\text { acorde con las } \\
\text { caracteristicas del } \\
\text { mismo. }\end{array}$ & $\begin{array}{l}\text { Realizar un } \\
\text { análisis a la } \\
\text { planeación } \\
\text { estratégica }\end{array}$ & $\begin{array}{l}\text { - Crear una misión, visión, objetivos } \\
\text { organizaciones y valores organizacionales } \\
\text { del establecimiento. } \\
\text { - Crear políticas de calidad. } \\
\text { - Estructurar la organización. } \\
\text { - Crear perfiles de cargos y capacitación } \\
\text { dentro del bar. } \\
\text { - Generar estrategias de reclutamiento. } \\
\text { - Generar estrategias de funcionamiento } \\
\text { intemo. } \\
\text { - Crear procesos de mejoramiento mediante } \\
\text { la lista de chequeo y el ciclo Deming. }\end{array}$ & $\begin{array}{l}\text { - El establecimiento } \\
\text { tiene un marco } \\
\text { estratégico en el cual } \\
\text { funcionar. }\end{array}$ \\
\hline $\begin{array}{l}\text { Definir aspectos } \\
\text { de formalización } \\
\text { empresarial. }\end{array}$ & $\begin{array}{l}\text { El establecimiento } \\
\text { conoce la } \\
\text { documentación } \\
\text { necesaria para } \\
\text { funcionar de } \\
\text { manera correcta. }\end{array}$ & \begin{tabular}{|l|} 
Realizar un \\
estudio legal
\end{tabular} & $\begin{array}{l}\text { - Constitución legal empresarial. } \\
\text { - Acondicionar el marco jurídico al } \\
\text { establecimiento. } \\
\text { - Documentar y acondicionar el marco legal } \\
\text { del sector económico de los bares. }\end{array}$ & $\begin{array}{l}\text { - El establecimiento } \\
\text { conoce del marco } \\
\text { legal y acondiciona el } \\
\text { establecimiento para } \\
\text { el mismo. }\end{array}$ \\
\hline
\end{tabular}

Fuente. El Autor

\section{CONCLUSIÓN}

Las conclusiones del estudio contemplan cada ítem realizado, cada hallazgo y cada solución generada, desde determinar el segmento del mercado, hasta la generación de estrategias de negocio. Éstas últimas son parte del compromiso adquirido por cada establecimiento. En primera instancia, se destaca que un correcto estudio de mercados determina gran parte de la viabilidad de un establecimiento. La contratación de una agencia de mercados, o la realización de un estudio propio por parte del establecimiento, debe determinar cómo atacar el nicho del mercado, al cual un bar pretenda apuntar.

El estudio técnico concluye que, gran parte de la problemática de un establecimiento, inicia en la escogencia de un local que, algunas veces, no cuenta con aspectos normativos básicos. La investigación recomienda generar, en prime- ra, instancia los requerimientos mínimos exigidos. Dependiendo de ellos, se toman decisiones para la localización del bar mismo y su correcta adecuación. Así mismo, mantener un contacto directo con las entidades rectoras, las cuales proporcionarán al bar los criterios para tener cierta estabilidad y mejorar su perdurabilidad. El estudio administrativo concluye la obligación de crear aspectos administrativos, para generar en primera instancia una visión realista del establecimiento. A su vez, una misión y unos objetivos claros y accesibles, en los que se involucren todos los elementos del bar, así como dar claridad a los aspectos organizacionales del establecimiento.

La investigación recomienda delimitar el alcance del establecimiento y adecuar las mejoras, de acuerdo al crecimiento y requerimientos del mismo. Este proyecto consiste en un diseño y no en un desarrollo, por lo que queda abierto para una futura investigación y su posible aplicación en un bar. 


\section{REFERENCIAS}

ASOBARES. Asociación de Empresarios de Bares. Disponible en: http://www.asobares. org/asobares.php. [Citado: 10 de Mayo de 2013].

Bacal, R. (2002) "Consiga el máximo rendimiento de su equipo" Madrid., Mcgraw hill.

Botero, L. (2009) "Indicadores de clientes". 6 de octubre. [Citado 20 de julio de 2014]. Disponible en internet: http://www. degerencia.com/articulo/indicadores-de-clientes-cuales-son

CERTICAMARA. Cámara de Comercio de Bogotá. [en línea] [Citado 20 de agosto de 2014]. Disponible en internet: http://camara.ccb. org.co/contenido/categoria. aspx?catID=106

Chiavenato, Idalberto, (2009), "Gestión del Talento
Humano". Ed McGraw-Hill, Tercera Edición.

CONCEJO DE BOGOTÁ D.C. Acuerdo 20 de 1995. [Citado 23 de octubre de 2014]. Disponible en internet: http://www. alcaldiabogota.gov.co/sisjur/ normas/Norma1.jsp?i=2052

Córdoba Padilla, Marcial (2006), "'Formulación y Evaluación de Proyectos". Ecoe Ediciones. Bogotá.

Díaz, S. (2010) "Ministerio de industria y turismo. Manual para la elaboración de planes de negocios". Punto aparte editores. Bogotá.

FONDO DE PREVENCIÓN Y ATENCIÓN A EMERGENCIAS. ¿Quiénes somos?. [Citado 6 junio de 2014]. Disponible en internet: http://intranet. fopae.gov.co/portal/page/portal/FOPAE_V2/Quienes $\% 20$ Somos
Hernández, R. (1997) "Metodología de la investigación". Ed Mcgraw-hill, 1997.

Rosell, J. (2013) "Gestión del bar y cafetería. Estrategias de éxito para su creación, organización y control". $1^{a}$ edición, ideas propias editorial, Vigo.

Sallenave, J. (1991) "La planificación estratégica". Segunda edición, México., 1991. [en línea] [citado 1 de octubre de 2014] disponible en internet: <http://www.gestiopolis. com/recursos2/documentos/fuIldocs/mar/planmktkarim.htm>

Soriano, C. (2013) "Pequeñas y medianas empresas, pyme 11-2005". Disponible en internet: <http://www.gestiopolis.com/canales5/emp/ochentapy.htm>. [consultado: 10 de mayo de 2013] 